\title{
Auftaktveranstaltung ventao e. V.: Vom „Lernen und Helfen“ zum entwicklungspolitischen Lern- und Austauschdienst ${ }^{1}$
}

\author{
Anette Schwitzke \\ Referentin für Qualitätsentwicklung und pädagogische Begleitung | ventao e.V., Berlin \\ anette.schwitzke@ventao.org \\ Jan Wenzel \\ Geschäftsführer | ventao e.V., Berlin | jan.wenzel@ventao.org
}

Mehr als 60 Interessierte nahmen an der Auftaktveranstaltung des neuen weltwärts-Verbundes „Verein entwicklungspolitischer Austauschorganisationen e. V.“ - kurz ventao teil. Die Teilnehmer_innen waren Vertreter_innen von Entsendeund Aufnahmeorganisationen des weltwärts-Programms, Kolleg_innen aus anderen Verbünden, Mitarbeiter_innen des Bundesministeriums für wirtschaftliche Zusammenarbeit und Entwicklung (BMZ), der Engagement Global gGmbH, der Stiftung Nord-Süd-Brücken sowie einige fachlich Interessierte.

Dem Verbund ventao haben sich 37 Organisationen angeschlossen, die im Rahmen des weltwärts-Programms entwicklungspolitische Freiwilligendienste organisieren. Die Mitgliedsorganisationen stehen für circa 500 Entsendungen und Aufnahmen von Freiwilligen. Sie verstehen Freiwilligendienst als entwicklungspolitischen Lerndienst für die Freiwilligen und als Instrument zur Stärkung von Partnerschaften zwischen Organisationen der Zivilgesellschaft in Deutschland und den Partnerländern.

Jan Wenzel, Geschäftsführer von ventao, gab den Teilnehmer_innen einen Blick auf die Arbeit des Vereins. Er betonte, dass sich das weltwärts-Programm und die im Kontext dieses Programms engagierten Akteure in den vergangenen Jahren erheblich weiter entwickelt haben. Der entwicklungspolitische Freiwilligendienst ist so zu einer beachtlichen Säule der entwicklungspolitischen Bildungsarbeit geworden. Ein ganz wesentlicher Schritt zu einem modernen Verständnis von entwicklungspolitischem Lernen sei dabei die Einführung der Süd-Nord-Komponente gewesen, die nunmehr ein wesentlicher Programmteil werden soll.

Er wies aber auch auf Herausforderungen hin. So stelle die Implementierung des weltwärts-Qualitätssystems mit Blick auf die zur Verfügung stehende ehrenamtliche aber auch hauptamtliche Zeit in den Organisationen eine enorme

1 Die Auftaktveranstaltung des Vereins entwicklungspolitischer Austauschorganisationen ventao e.V. fand am 11. Februar 2014 in Berlin statt. 
Herausforderung dar. Der Verbund werde die Mitgliedsorganisationen nach Kräften in diesem Prozess unterstützen. Gemeinsam habe man sich dafür aber auch vorgenommen, dass die Mehrarbeit für die neue „Qualitätsoffensive“ nicht zu Lasten der Arbeit mit den Freiwilligen, den Partner_innen und den entwicklungspolitischen Inhalten gehen darf.

Schließlich ging Jan Wenzel auf die durch den Koalitionsvertrag von CDU/CSU und SPD aufgeworfene Diskussion über die Verortung der Zuständigkeit für die internationalen Freiwilligendienste ein („Harmonisierung“). Die Mitgliedsorganisationen vertreten in dieser Frage die Position, dass die Förderung entwicklungspolitischer Freiwilligendienste über das weltwärts-Programm auch weiterhin beim BMZ liegen müsse. Sie verstehen den entwicklungspolitischen Freiwilligendienst als ein Instrument der entwicklungspolitischen Arbeit, der auch die Arbeit mit den Partner_innen im Globalen Süden begleiten soll. Nur mit einem Verbleib beim BMZ bleibt eine Kohärenz mit anderen entwicklungspolitischen Programmen erhalten.

Auf zwei Podien mit Vertreter_innen aus Praxis, Wissenschaft und aus dem für weltwärts zuständigen BMZ-Referat „Bürgerschaftliches Engagement“ wurde im Anschluss erörtert, wie weltwärts geförderte Freiwilligendienste sich auf verschiedene Organisationen ausgewirkt haben und welchen konkreten Herausforderungen sich das Programm als Ganzes, aber auch die einzelnen Organisationen, stellen müssen.

Stefanie Kleppe, weltwärts-Koordinatorin des Eine-Welt-Haus Jena, berichtete, wie die Freiwilligen und insbesondere die Rückkehrer_innen einen Generationenwechsel in der Städtepartnerschaft zwischen Jena und San Marcos in Nicaragua befördern. Sowohl in Jena als auch in San Marcos engagieren sich durch die Freiwilligendienste mehr junge Leute und bringen neue Ideen in die bildungspolitischen und sozialen Projekte ein. Mehr als die Hälfte der Rückkehrer_innen engagiert sich nach dem Freiwilligendienst für das Eine-Welt-Haus. Das Aufeinandertreffen der Generationen sei dabei zwar nicht immer reibungslos und einfach. Insgesamt sei aber festzustellen, dass die Vereine und die gemeinsamen Projekte von dem Engagement profitierten.

Maximilian Vogel vom Verein Zugvögel Interkultureller Süd-Nord-Austausch e.V., der sich in den vergangenen Jahren sehr stark dafür gemacht hat, dass die SüdNord-Komponente eingeführt wird, zeigte sich einerseits erfreut darüber, dass nun ein Anfang dafür gemacht sei, dass erste Freiwillige gefördert nach Deutschland kommen können. Gleichzeitig wies er aber daraufhin, dass damit erst der Auftakt für einen intensiveren Dialog und partnerschaftlichen Austausch über verschiedene Perspektiven auf Machtstrukturen und Entwicklung gemacht sei.

Birgit Pickel, BMZ-Referatsleiterin, bestätigte, dass sich das Programm seit seiner Einführung inhaltlich sehr stark weiterentwickelt habe. Die Orientierung auf 
realistische Beiträge und Wirkungen des Freiwilligendienstes auf der Ebene der Partnerorganisationen, aber auch auf das Lernen und das Engagement der Freiwilligen, sei in den vergangenen Jahren sehr wichtig gewesen. Dabei habe man viele Anregungen von den Partner_innen in der Zivilgesellschaft, aber auch aus der Evaluierung aufgenommen, die in die Überarbeitung der Förderbedingungen des Programms eingeflossen seien. Auch von BMZ-Seite freue man sich nun auf neue Impulse aus der Süd-Nord-Komponente. Auf Ebene der Programmsteuerung strebe das BMZ an, weiterhin für große und kleine Akteure offen zu sein, dabei aber einen gemeinsamen Qualitätsstandard abzusichern. Im Hinblick auf die genannte Diskussion der Zuständigkeiten verschiedener Ministerien für internationale Freiwilligendienste wies Birgit Pickel darauf hin, dass weltwärts für das BMZ ein zentrales Instrument sei, um entwicklungspolitische Bildungsarbeit und entwicklungspolitisches Engagement zu fördern. Daher setze sich ihr Haus dafür ein, dass die Zuständigkeit weiterhin im BMZ verankert bliebe.

Auf dem zweiten Podium beschäftigten sich die Teilnehmer_innen mit einigen Herausforderungen, denen sich weltwärts trotz der Entwicklung der vergangenen Jahre stellen muss.

Carola Blendermann, Geschäftsführerin von Sage Net e.V., berichtete von einer Studie, die aus Sicht der Partner_innen und der Freiwilligen Kriterien für einen gelungenen Freiwilligendienst erarbeitet hat. ${ }^{2}$ Aus einer qualitativen Evaluation, die Sage Net 2011 durchgeführt hat, hat der Verein mit seinen Partnern viele Rückschlüsse für die weitere Arbeit gezogen. Man habe sich dadurch intensiv mit der oft „überambitionierten Haltung“ von Freiwilligen beschäftigt und analysiert, wie sich die dem gegenüberstehende Haltung der Partnerorganisationen auf diese Motivationen auswirkt. Die Erkenntnisse dieser qualitativen Befragungen und der Auswertung sind unter anderem in mehrere Dialogveranstaltungen, aber auch ganz konkret in die Begleitung der Freiwilligen und die Entwicklung einer gemeinsamen Sage Net Policy eingeflossen. Resümierend verwies sie darauf, dass solche Studien und Evaluierungen sehr aufwendig im Hinblick auf die finanziellen und personellen Ressourcen seien. Sage Net würde aber die Verfolgung dieser Wirkungsbeobachtung gerne weiter entwickeln, um in der Praxis auf diesen Erkenntnissen aufbauen zu können.

Barbara Schütz vom Welthaus Bielefeld beleuchtete die Herausforderungen für eine kontinuierliche und bessere Einbindung der Partner_innen in der Programmgestaltung. Bereits zu Beginn ihres nachdenklichen Statements bedauerte sie, dass es z.B. nicht immer möglich sei Partner_innen selbst zu Wort kommen zu lassen,

2 Die Zusammenfassung der Studie mit dem Titel "Research based recommendations for enhancing mutual satisfaction and success in international voluntary service" finden Sie hier: http://sagenetger.jghinternet. co.za/backend/media/1010493461754.pdf 
wie es zu diesem Thema auf einer solchen Veranstaltung wünschenswert, vom Aufwand aber nicht verhältnismäßig gewesen wäre. Sie warnte auch vor der Gefahr, die eine „Scheinpartizipation“ mit sich bringen würde. Trotzdem sei die Förderung des Dialogs, das sich gegenseitig Ernst nehmen und Suchen nach Möglichkeiten der Einbindung in relevante Entscheidungen bei weitem nicht ausgereizt. So sei es elementar, dass wichtige Dokumente den Partnern_innen zugänglich gemacht würden und Diskussionen z. B. über Qualitätskriterien oder Süd-Nord-Austausch mit den Partner_innen geführt würden. Sie sprach sich dafür aus, hier weiter zu denken und den Dialog zu führen und nicht mit dem Argument zu bremsen, dass das nicht praktikabel sei.

Kristina Kontzi vom Berliner Verein glokal e.V. hat im Rahmen einer Doktorarbeit die Programmdokumente von weltwärts analysiert. Sie konstatierte, dass dort trotz weitgehender Überarbeitung noch immer Haltungen repräsentiert werden, die eine Überlegenheit des Nordens gegenüber dem Süden widerspiegelten. Dies äußere sich zum Beispiel an der Darstellung von Freiwilligen aus Deutschland. Obwohl diese in aller Regel kaum besondere Qualifikationen mitbrächten, würden sie in der Außendarstellung häufig als „Changemaker“ dargestellt. Bei den Partnerorganisationen aus dem Süden stünden dagegen in aller Regel die Defizite im Vordergrund. Die Freiwilligen würden aktiv, engagiert und wissend dargestellt, wogegen die Partner_innen als gleichgültig und wenig partizipativ erschienen. Dadurch setze sich eine koloniale Blickweise fort. Befördert werden sollte aus Sicht von Kristina Kontzi eine Ausrichtung auf das Ziel, die Freiwilligen zu kritischen „Global Citizens“ auszubilden. Dafür sei das entwicklungspolitische Engagement der Freiwilligen im Ausland nicht immer zwingend erforderlich.

Die Veranstaltung schloss mit einem würdigenden Blick auf die Servicestelle weltwärts der Stiftung Nord-Süd-Brücken durch Walter Hättig, Geschäftsführer der Stiftung Nord-Süd-Brücken und Birgit Pickel (BMZ). Die Servicestelle war fünfeinhalb Jahre lang unter der Führung von Jan Wenzel wertvoller Impulsgeber für die weltwärts- Programmentwicklung. Jan Wenzel bedankte sich wiederum für die Unterstützung durch die Stiftung Nord-Süd-Brücken und bei den Akteuren aus den Entsendeorganisationen und in den Gremien des Programms für die konstruktive Zusammenarbeit. Die servicestelle weltwärts geht nun, mit Jan Wenzel als Leitung, in den neuen Verein entwicklungspolitischer Austauschorganisationen e.V.über. 